\title{
DATA TRANSFER PROTOCOLS FOR BUSINESS SOCIAL NETWORKING
}

\author{
Venko Andonov, Valentin Kisimov, Tsvetana Stoyanova and Nikolay Sterev \\ University of National and World Economy, Sofia, Bulgaria
}

\begin{abstract}
The next generation technologies are based on the smart communication between different users and product and services providers. Thus, the sharing economy could be explained as a process of continuous exchange of the information and data packages between different stakeholders. In these terms, the global economy is described as a global computer network and unstoppable data transfer between each two elements - global Business Social Network (BSN).

As the smooth Social Networking needs RULES for data transfer, the paper focuses not just on defining Business Social Networking requirements but on the main protocols for sharing information and for exchange data packages among the corporate participants of the BSN. Thus, the paper uses already defined three layers of the corporate BSNs as: Core layer of BSN, economic layer of BSN; social layer of BSN. 1.) Core Layer of Business Social Network (BSN) defines the elements for logical protocol of data transfer that are similar to Traditional Social Network (TSN), but works outside a single corporation. Core of the BSN is the cooperation, project specific teaming, and business and solution generation discussions inside in a corporate division from one side or between particular divisions from the other. 2.) Economic Layer of Business Social Network (BSN) express these elements of quantitative and qualitative indices of the business goals that are based on the function of the BSN. These indices are very important for the functioning of the contemporary sharing corporation and they could be neglected in the traditional corporations as: time saving; information partaking; social group participation and etc. 3.) Social Layer of Business Social Network (BSN) gives the image of BSN as "talking structure" that allows to the users to exchange their opinions, emotions and behavior that are usually forbidden for expression at the traditional corporate networking.

The structure of the paper covers the next 4 elements: Introduction: the definitions of the sharing economy and digital platform's solutions are given as well as the explanation of the framework of BSN is presented. 1. Business-to-Business (B2B) communication protocols: the architecture design of the BSN is explained and the main sets of communication protocols are given. Technical solution for the main B2B objects and their integration in BSN are proposed. 2. Web Social BSN protocols: these protocols are elements of the interaction of the partners BSNs and they are specific in digital era. They allow social network be accepted as a interoperable service that is decentralized and distributed across distinct BSN's actors. Conclusions: the main results of the research are summarized in given architecture solution for a B2B BSN with two business partners participating in a non-centralized, distributed social network. Some limitations of the Electronic Data Interchange (EDI) are also discussed.
\end{abstract}

\section{KEYWORDS}

Business Social Network (BSN), B2B, Cross- Corporate BSN, BSN Logical Protocol, Web Social Protocol

\section{INTRODUCTION}

The expansion of the social networking (SN) nowadays is based on the opportunities that SNs provide to people for establishing connections with colleague or friends or for developing new friendships by person-to-person communications [Roberts and Roach, 2009 in Barczyk C., D. Duncan, 2011]. The SNs development is based on introduction of new generation of social media as a set of Internet-based applications that allow free exchange of User Generated Content through them. [Kaplan and Haenlein, 2010 in Barczyk C., D. Duncan, 2011; Benkler 2006].

The advantages of this virtualization of the person-to-person and resp. business-to business (B2B) relations is based on the main characteristics of digital networks: non-technical users, social type of users, and establishment of interpersonal relations [Agarwal and Mital, 2009 in Barczyk C., D. Duncan, 2011]. The future digitalization of B2B relations are based on further development of web applications and services that could 
introduce substantial changes to communication between businesses and different stakeholders as social and business communities, and individuals (clients) [Siricharoen, 2012].

The business accepts the Business Social Networks as an opportunity for instant messaging that make business more and more interactive as well as an opportunity for real time revision and update of the given information through social media channels [NMC, 2007 in Siricharoen, 2012]

Furthermore, business network is defined as a set of interconnected business relationships [Anderson et al., 1994 in Škarpová and Grosová, 2015]. Thus, business social network (BSN) is a set of people, preferably with a broad array of experience and knowledge, to which an individual is connected and with which the individual is in periodic contact. Ideally, any member of this BSN represent different stakeholders [Byham, 2008].

Thus, the BSN is established on three different layers: social; economic and core layers (Figure 1.)

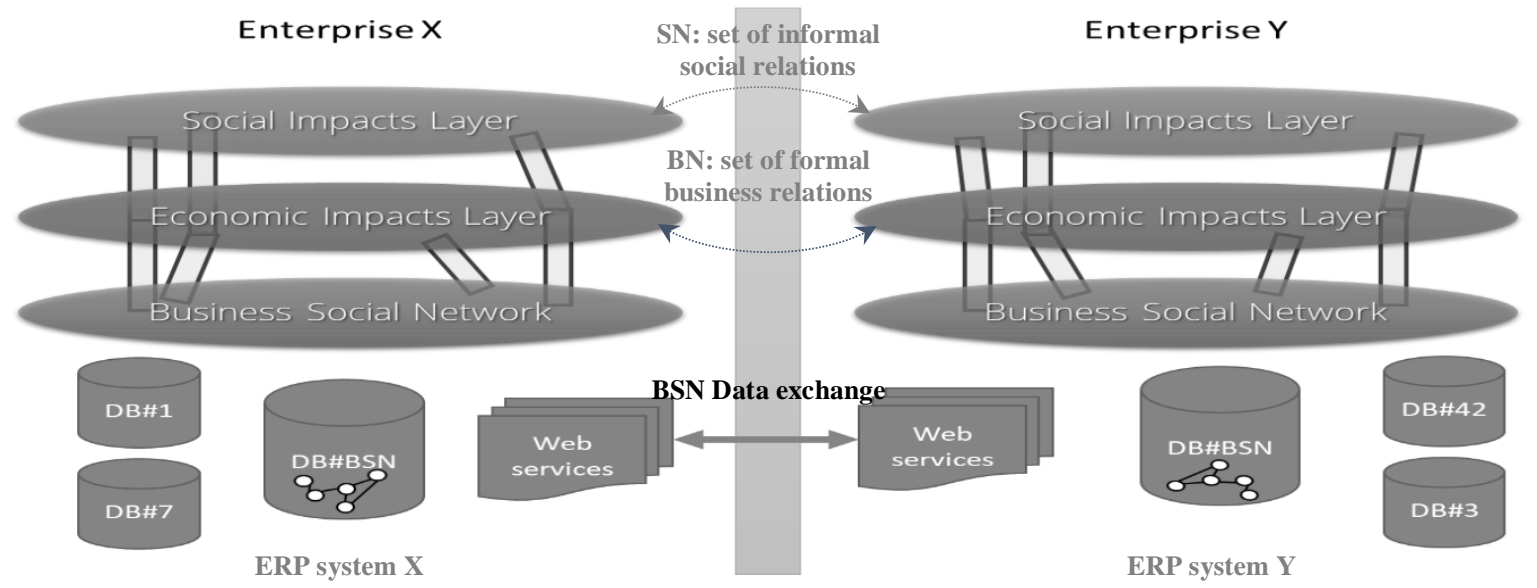

Source: Sterev, Kisimov, Stoyanova, Andonov, 2018a, 2018b

Figure 1. The data exchange for the B2B BSN communication through standard Internet-based web applications

The Business Social Network Core Layer defines the elements for logical protocol of data transfer:

- the objects, the relationships between them and how they are stored and processed, including the rules and constraints defined by the business requirements;

- the interlayer relationships between the defined objects and the indicators in the economic and social impact layers;

- the cross-business relationships between the objects defined as part of the communication protocol between any given business entities.

The abstract object types of the BSN core layer include:

- an AGENT - a user or automated process, participating in the Business Social Network; forms relationships with other Agents or other Content objects;

- a CONTENT ITEM - any object that might be shared in the BSN as part of the discussion and collaboration between the Agents; forms relationships with other Content items and different Agents;

- a DYNAMIC DATA STRUCTURE - contains a definition of a data structure that is populated manually or automatically during the collaboration and cross-teaming effort; forms relationships with other data structures, content items and agents;

- a GROUP - an object which unites multiple Agents, Content items and Dynamic data structures under a specific unique identity with its own permission structure; forms relationships with other Groups, Agents, Content items and Dynamic data structures.

In order to manage the digital data transfer among BSN, the architecture of the BSN Core layer follows these 5 principles:

- The network consists of multiple nodes (each business enterprise might have their own node).

- The nodes are dependent of each other only as much as defined in the security and privacy protocol configuration.

- There is no central authority that manages the whole network of multiple business entities - each node is feature-complete and is capable to function properly on its own. 
- The inclusion of a new node in the business network consists of two main steps: defining its own security and privacy configuration; each of the other nodes in the network should add the new node with their own proper security and privacy configuration.

- It is possible for a given node to participate in different business networks with different security and privacy configurations

\section{BUSINESS-TO-BUSINESS (B2B) COMMUNICATION PROTOCOLS}

As the BSN is distributed on multiple nodes, typically in separate business enterprises (the concrete implementation might vary), there is no central server that would encapsulate the whole network since such a network requires strict security and privacy rules as discussed above. Furthermore, a central authority would require a centralized management of the system, which would be a significant organizational and managerial overhead for a system that tries to improve and optimize the business processes and communication between separate business entities.

Our view of a B2B BSN is as a distributed system of multiple nodes without a central authority, which communicate with each other over a strict set of protocol rules in order to achieve information sharing within the bounds of the defined objects and relationships.

Physically, the nodes might be single servers, server clusters, cloud servers or any other solution that might fit the technological organization of a given business entity. Each person within the given business entity accesses only their own service, which, on the other hand, communicates with the other nodes in the predefined social network in order to perform a requested operation. This architecture allows for federated machine learning as well - the ability to train ML models on separate nodes without sharing the raw data.

\subsection{RPC - Remote Procedure Calls}

Historically, especially until the mid-90s, most computers interacted with each other using Remote Procedure Calls (RPC). This is client-server interaction model, where a client executes a procedure on a remote server in order to perform a specific operation and optionally receive results back [Baer, 2018].

The idea is to allow the developer to treat the procedures in the remote environment as if they were local, of course considering the network overhead and unreliability. Since it is unconstrained by the structure of data, highly specialized methods can be written for clients that request and retrieve exactly the information needed which can result in minimal network overhead and smaller payloads. The local and the remote systems are tightly coupled, the boundaries between the local and the remote code are lost [Baer, 2018]. This might lead to many types of difficulties in the management, maintenance and further development and this is one of the main reasons the standard RPC are considered obsolete nowadays.

- $\quad$ gRPC is a modernized alternative of the classical RPC approach. It is an open source, high performance RPC framework that can run in any environment. Initially, it was developed within Google for internal use. It can efficiently connect services (usually microservices) with pluggable support for load balancing, tracing, health checking and authentication. gRPC can be used also to connect devices, mobile applications and browsers to backend services. It is usually implemented for communication between services in a microservices style architecture; for connecting mobile devices and browser clients to backend services; for generating efficient client libraries [gRPC Network, 2019].

\subsection{Web Services}

- SOAP (Simple Object Access Protocol) is a protocol specification for XML based communication between applications (typically distributed). It has been created to address some of the practical problems and difficulties of RPC, by creating a well-structured foundation for complex web services. The enforceable contracts in the WSDL and WADL between the client and server guarantee predictable, type-safe results, and the WSDL can be used to generate documentation or to create integrations with IDEs and other tools. The most significant downside of SOAP is that it is very 
verbose and in practice, requires sophisticated tools in order to develop and debug systems based on it. Tools are necessary for testing, for inspection, parsing the data. There are still many systems operating with SOAP nowadays, but it is typically not recommended for new projects since it is considered impractical and too verbose [Baer, 2018].

- $\quad$ REST (Representational State Transfer), unlike SOAP, does not use client-server contracts, is much more concise and does not require special tools. It uses the HTTP (Hypertext Transfer Protocol) like verbs and URIs to reference and perform operations on a resource in some hierarchy of data. REST completely and explicitly changes API design from modeling interactions to modeling the data of a domain. Being fully resource-oriented when working with a REST API, the developer does not have to know anything about the implementation of the backend services.

REST services typically take multiple round trips between client and server to get enough data to render an application. This cascade of requests has devastating performance impacts, especially on mobile. Another problem with REST services is that the send way more information than is needed. There are a lot of projects that attempt to fix the shortcomings: HAL, Swagger/OpenAPI, OData, JSON API and more.

- In GraphQL, on the server, the data is described as a graph of interconnections, and the client asks specifically for the subset of the data that it needs [Baer, 2018]. This approach solved two of the most significant drawbacks of the classical REST services - generating too many requests and responses for related data entities, as well as transferring data that is irrelevant to the current context. Currently, it is considered to be the state-of-the-art approach for distributed applications communication, as it proposes solutions for the shortcomings of the earlier standards.

\subsection{Other Client-Server Protocols}

File Transfer Protocol (FTP) is a protocol for computers connected through the Internet (and other TCP/IP networks) to securely transfer files between each other [ArcESB, 2019]. FTP is used for many types of file transfer, for example, website updates and bulk file transfers. FTP is the most widely used file transfer protocol, but due to security issues, most organizations only use FTP for transferring files within the organization. The big drawbacks are that it doesn't encrypt data in transit and uses separate command and data channels [ArcESB, 2019].

Often, businesses connecting through FTP use a virtual private network (VPN), which provides an extra layer of security around transactions.

- $\quad$ SFTP is an enhanced version of FTP with a different security layer - SSH. This security layer encrypts the messages while in transit and decrypts the messages upon arrival. SFTP requires the server to authenticate the client computer. All commands and data are encrypted to prevent passwords and other sensitive information from being exposed to the network in plain text. Unlike FTP, SFTP does protect data during transmission and doesn't use separate command and data channels - it transfers data and commands in formatted packets with one secure connection.

- $\quad$ FTPS is also an alternative approach - FTP over SSL/TSL - adds a secure encryption layer (Secure Sockets Layer) around the FTP protocol to secure the commands and data transferred between client and server.

- Odette File Transfer Protocol (OFTP) is a communications technology built specifically for B2B document exchange. Unlike the basic FTP protocols, it can provide digitally signed electronic delivery receipts and provides multiple security features - transport security with TLS, file encryption, partner authentication [ArcESB, 2019; Odette, 2019].

\section{SOCIAL NETWORKING PROTOCOLS}

\subsection{Definition of Social Networking Protocols}

A distributed (also called federated) social network is an interoperable service that is decentralized and distributed across distinct entities. A social website participating in a distributed social network is interoperable with the other sites in the same network and is in federation with them. Communication among them is 
conducted over social networking protocols [Esguerra, 2011]. There have been numerous different standards over the years since this is a popular issue in the public space because of the privacy concerns with the centralized social networks (like Facebook, Twitter and others).

The Social Web Protocols are a collection of standards which enable various aspects of decentralized social interaction on the Web [W3C, 2017]. People and the content they create are the core components of the social web; they make up the so called "social graph". The Social Web Protocols describe a way in which people can: create, update and delete social content; connect with other people by subscribing to their content; interact with other peoples' content; be notified when other people interact with their content. regardless of what that content is or where it is stored [W3C, 2017]. These components are core building blocks for interoperable decentralized social systems. Each of these components can be implemented and extended to meet domain-specific requirements, such in the case of distributed B2B communication.

The Social Web Protocols define the following structures: Social Data Syntax - A JSON-based syntax to allow the transfer of social information; Social API - defines a specification for a client-side API that lets developers embed and format third party information; Federation Protocol - allows the federation of activity-based status updates and other data (such as profile information) between heterogeneous social systems [W3C, 2017].

- ActivityStreams 2.0 is the recommended syntax and vocabulary for social data. It represents content and interactions as Objects and Activities. The ActivityStreams vocabulary defines a finite set of common Object and Activity types and properties, as well as an extension mechanism for applications which want to expand on or specialize these [W3C, 2017].

- ActivityPub provides two layers: a server to server federation protocol (so decentralized websites can share information); a client to server protocol (so users, including real-world users, bots, and other automated processes, can communicate with ActivityPub using their accounts on servers, from a phone or desktop or web application) [W3C, 2018]. ActivityPub uses ActivityStreams for its vocabulary.

ActivityStreams includes all the common terms that are typically needed to represent all the activities and content flowing around a social network. If this vocabulary is not enough for a given use case, it is possible to use JSON-LD to extend it.

ActivityPub uses authentication for two purposes; first, to authenticate clients to servers, and secondly in federated implementations to authenticate servers to each other. ActivityPub clients authenticate against a server using OAuth 2.0 bearer tokens. Server to server federation is authenticated using HTTP Signatures in conjunction with the signing key from the actor's publicKey field. The keyId should link to the actor so that the publicKey field can be retrieved. At minimum, the digest field should be included in the set of headers being signed. For most forms of delivery, HTTP Signatures is enough. However, some objects may be passed around the network, whether being shared, forwarded, or otherwise referenced at a future time. In this case it is important to be able to verify that the actors specified in the actor and attributedTo fields really did author the objects they are being claimed to have authored. In such a case we need to be able to verify the integrity of the object being passed around, and so we must sign the object itself. For this we use Linked Data Signatures to sign the object with the publicKey of the actor that is the actor or attributed to of the object being passed around the network.

Servers should not trust client submitted content, and federated servers also should not trust content received from a server other than the content's origin without some form of verification. They should also implement protections against denial-of-service attacks from other servers. This can be done using rate limiting mechanisms or algorithms for anomaly detection. Servers should be especially careful to implement this protection around activities that involve side effects.

Working implementations of these standards are Mastodon ${ }^{1}$ and Friendi.ca ${ }^{2}$, which are both free and open source software products for distributed, decentralized social networking.

\footnotetext{
${ }^{1}$ https://joinmastodon.org/

${ }^{2}$ https://friendi.ca/
} 


\subsection{Security and Privacy Configuration of a B2B-BSN: Node and Network}

The security and privacy configuration of a node consists of a strictly formatted, machine-readable description of the permissions of each object type related to a specific other connected node.

Zot is a web standard and framework for implementing secure decentralized communications and services [Hubzilla, 2019]. The main difference with other communication standards is that it is built on top of a decentralized identity and authentication framework. The authentication is similar to OpenID conceptually but is insulated from DNS-based identities. Remote authentication should be silent and invisible to the end-user of the system. This provides a mechanism for internet scale distributed access control which is unobtrusive [Hubzilla, 2019]. Zot supports a wide array of background services in the grid, from friend suggestions to directory services. New content and data updates are propagated in the background between the nodes in the network according to access control lists and permissions specified by both sender and receiver channels.

Zot's identity layer allows each of the business partners to provide granular permissions to any content - social or source business document - and these permissions extend across the partner network. Access can be granted or denied for any resource, to any channel, or any group of channels; anywhere within the B2B network.

\section{B2B - BSN SOLUTION AND CONCLUSION}

Summarizing the main rules for architecture and protocols organization of B2B Business social networking the main web solution for BSN with two business partners participating in a non-centralized, distributed social network is presented on Figure 2.

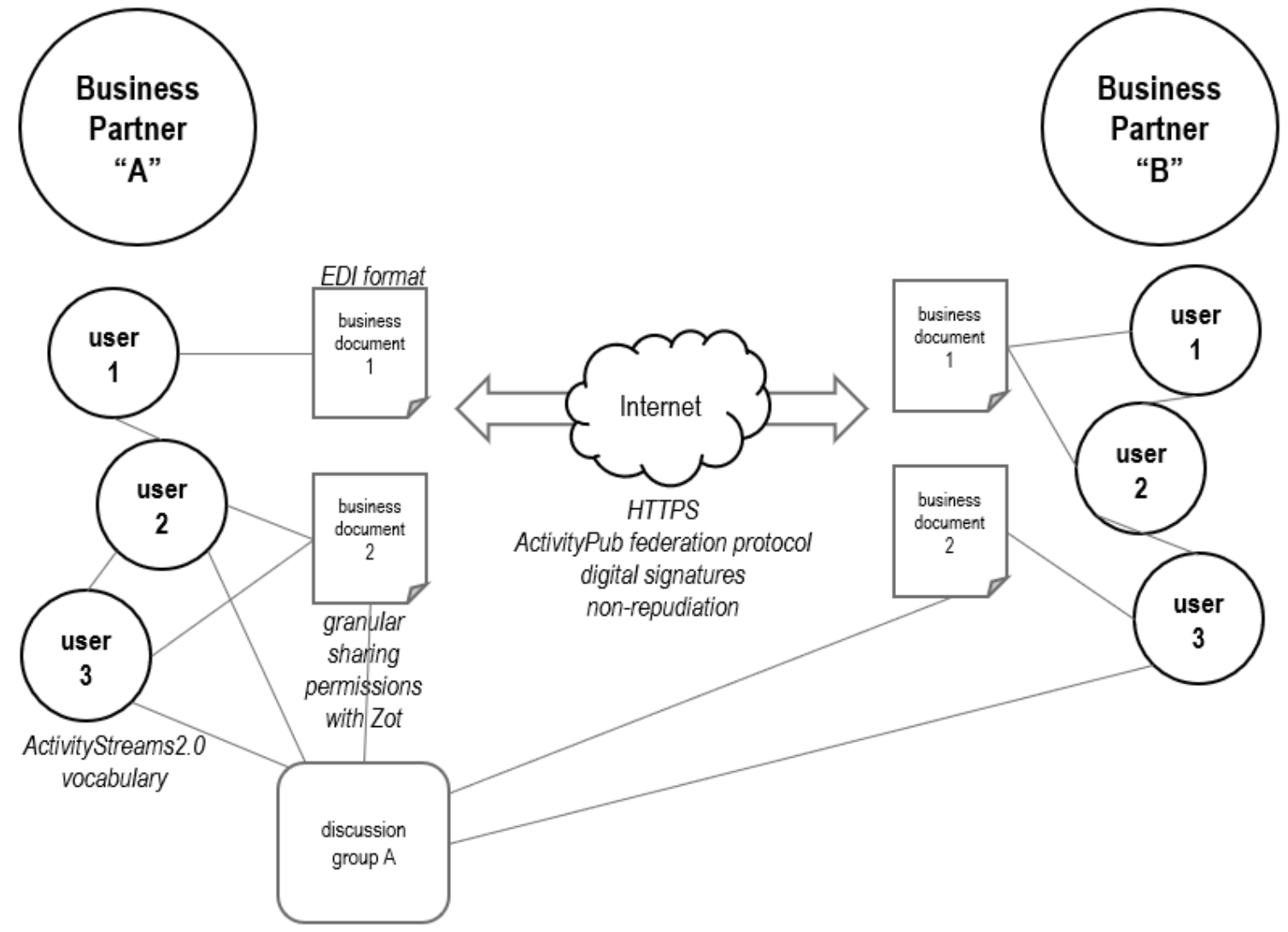

Figure 2. B2B - BSN Web solution 
The given on Figure 2 data transfer between 2 business enterprises in web based BSN needs to explain furthermore main data transfer formats.

Electronic Data Interchange (EDI) is a communications technology and a group of standards used to exchange business documents and information between organizations. It can handle multiple types of data, including but not limited to orders, invoices, quotes, shipment data, insurance information [ArcESB, 2019]. The goal of EDI is to replace the manual, often paper-based, B2B communications, such as postal mail, fax and email. Documents are sent directly from the sender's computer system to the receiver's system. These systems might be ERP-s, CRM-s, SCM-s or any type of business information system. There are a variety of EDI standards for various industries, regions and use cases - each with different versions, so EDI partners must use the same standard and version. Popular standards include: ANSI X12 in the United States, UN/EDIFACT globally and industry-specific standards, such as HIPAA (health insurance in the U.S.), HL7 (for international healthcare data exchange), EANCOM (in the retail sector), ROSETTANET (electronics) and others. This diversity creates a significant implementation difficulty as the software developers should cover multiple protocols and the possibility of conversion. Typical documents transferred through EDI are: orders, quotes, invoices, shipping notices, product information, pricing and other depending on the specific industry.

EDI protocols are the different descriptions of data and communication formats that might be used to transfer data securely from one company to their partner. Each protocol reflects a set of universal guidelines that dictate how the data is encrypted and configured while in transit [3]. In direct EDI, also known as point-to-point EDI, each business partner connects directly to the other. An alternative approach is an indirect connectivity - using the so-called Value-Added Networks (VAN), which act as middlemen for the data exchange and help with the setup and the conversions between the different protocols.

- The AS1 (Applicability Statement 1) EDI Protocol describes methods to use the e-mail protocols to transfer data in a secure and reliable way. The AS1 protocol is based on SMTP and S/MIME. It was the first AS protocol developed and uses signing, encryption and message delivery notifications conventions. Files are sent as attachments in a specially coded S/MIME email message [ArcESB, 2019].

- $\quad \mathbf{A S 2}$ is the second-generation EDI protocol - it is the most widely used EDI standard nowadays [ArcESB, 2019]. AS2 defined how to transfer the business documents over the Internet using the HTTP (more specifically - HTTPS). An envelope of the transferred data is created so it can be sent securely - using digital certificates, signatures and encryption. It also supports signed receipts to ensure that the business partner actually received the message. Since the AS2 protocol operates over ordinary HTTP, it can easily pass through firewalls and utilize optional transport level SSL encryption and HTTP authentication. The digital certificates are used to encrypt the documents sent over the public Internet, with digital signatures to guarantee integrity and non-repudiation [Ruparathna, 2017].

- $\quad$ AS3 is built on File Transfer Protocol (FTP). It also enables the business partners to securely send and receive documents. AS3 enables push and pull FTP capabilities, similar to setups in asynchronous and bi-synchronous environments. It is not used as much as AS2 and AS4. As discussed above, the standard FTP can be replaced by FTPS or SFTP for a more secure data transfer.

- AS4 is a web-services (more specifically SOAP) update to AS2, built largely on the OASIS ebMS framework. The Message Service Specification (ebMS) describes a communication-neutral mechanism that must be implemented in order to exchange business documents [OASIS 2019, ArcESB 2019]. ebMS is built as an extension on top of the SOAP with Attachments specification. The SOAP message contains the metadata required to exchange the business document in a secure and reliable manner, while the business payload is attached to the SOAP message [OASIS 2019]. AS4 can transport any type of document as defined by the EDI standard. It maintains the integrity and the confidentiality of the business data by using the WS-Security standard. It provides password authentication, digital signatures and encryption; transport security with TLS; authenticity verification of the sender; ensures messages are unaltered in transit; provides non-repudiation. 


\section{ACKNOWLEDGEMENT}

The paper is prepared as a part of the research activities under the project "Knowledge Economy based on business platforms and enterprises' networks" (DN 05/17/2016), financed by the Bulgarian National Science Fund.

\section{REFERENCES}

Agarwal, S., Mital, M., 2009, An exploratory study of Indian university students ${ }^{\text {ee }}$ use of social networking web sites: Implications for the workplace, Business Communication Quarterly, 105, March. 2009

Anderson, C. J., Håkansson, H., Johanson, J., 1994, Dyadic Business Relationships Within a Business Network Context. Journal of Marketing, 58 (4) / 1994, 1-15.

ArcESB, 2019, What is EDI? The Complete Guide to Electronic Data Interchange, [Online]. Available: https://www.arcesb.com/resources/edi/. [Accessed 2019].

Baer E., 2018, A GraphQL Primer: Why We Need A New Kind Of API (Part 1), Smashing Magazine, January 2018. [Online]. Available: https://www.smashingmagazine.com/2018/01/graphql-primer-new-api-part-1/.

Barczyk C., D. Duncan, 2011, Social Networking Media as a Tool for Teaching Business Administration Courses, International Journal of Humanities and Social Science, Vol. 1 No. 17 / 2001

Benkler Y., 2006, The wealth of networks: how social production transforms markets and freedom, New Haven, Conn: Yale University Press, pp.528.

Byham W.C., 2008, Business Networking: A Necessary Third Millennium Skill, White Paper Development Dimensions International, Inc., https://www.ddiworld.com/ddi/media/white-papers/BusinessNetworkingbyham_wp_ddi.pdf

Esguerra E. R., 2011, An Introduction to the Federated Social Network, Electronic Frontier Foundation (EFF), 2011. [Online]. Available: https://www.eff.org/deeplinks/2011/03/introduction-distributed-social-network.

gRPC Network, 2019, About gRPC, [Online]. Available: https://grpc.io/about/. [Accessed 2019].

Hoffman C., 2019, How HTTP/3 and QUIC Will Speed Up Your Web Browsing," How-To-Geek, September 2019. [Online]. Available: https://www.howtogeek.com/442047/how-http3-and-quic-will-speed-up-your-web-browsing/.

Hubzilla, 2019, Zot Protocol, [Online]. Available: https://zotlabs.org/help/en/developer/zot_protocol. [Accessed 2019].

Kaplan, A. and Haenlein, M., 2010, Users of the world, unite! The challenges and opportunities of social media, Business Horizons, 53(1), pp. 59-68.

OASIS, 2019, About ebXML," [Online]. Available: http://www.ebxml.org/geninfo.htm.

Odette, 2019, OFTP2, [Online]. Available: https://www.odette.org/services/oftp2 [Accessed 2019].

Roberts, S. and Roach, T., 2009, Social networking web sites and human resource personnel: Suggestions for job searches, Business Communication Quarterly, pp. 110-114.

Ruparathna R., 2017, AS2 Protocol for Business Data Interchange via HTTP, DZone Devada Media, 2017. [Online]. Available: https://dzone.com/articles/as2-protocol-for-business-data-interchange-via-htt.

Siricharoen W. V., 2012, Social Media, how does it Work for Business? International Journal of Innovation, Management and Technology, Vol. 3, No. 4, August 2012

Škarpová L., G.Stanislava, 2015, The Application of Business Network Approach for Small and Medium Enterprises (SME) with regard to their Buying Behavior, Journal of Competitiveness, Vol. 7, Issue 3, pp. 62-74, September 2015

Sterev, Kisimov, Stoyanova, Andonov, 2018a, Multidimencial Framework for Crosscorporate Business Social Network (BSN), Proceedings of 11th IADIS International Conference on Information Systems (IS 2018), Lisbon, Portugal 14- 16 April 2018

Sterev, Kisimov, Stoyanova, Andonov, 2018b, INDUSTRY 4.0 NEEDS of Business Social Network (BSN)), Proceedings of ICEIRD 2018, Doha, Qatar, 28-30 Oct 2018

The New Media Consortium (NMC), 2007, The "Third place," and The Evolution of Communication. [Online]. Available: http://creativecommons.org/licenses/by-sa/3.0/us/.

W3C, 2017, Social Web Protocols, World Wide Web Consortium (W3C), December 2017. [Online]. Available: https://www.w3.org/TR/social-web-protocols/.

W3C, 2018, ActivityPub, World Wide Web Consortium (W3C), January 2018. [Online]. Available: https://www.w3.org/TR/activitypub/. 